\title{
Comment to the paper: extended endoscopic endonasal approach to the suprasellar parachiasmatic cisterns: anatomic study by Abuzayed Bashar
}

\author{
Paolo Cappabianca
}

Received: 12 June 2010 /Accepted: 12 June 2010/Published online: 30 June 2010

(C) Springer-Verlag 2010

This is not the first, and will not be the last, good anatomical study coming from the Turkish Neurosurgical Schools that has always demonstrated the appreciation and respect they attribute to anatomical dissection as the basis of any

P. Cappabianca $(\bowtie)$

Università degli Studi di Napoli Federico II,

Division of Neurosurgery,

Via Sergio Pansini 5,

80131 Naples, Italy

e-mail: paolo.cappabianca@unina.it neurosurgical technique. The present report is appropriate and accurate, and complements previous observations on the same area, adding an insight on the relevant cisternal spaces. This is even more welcomed because the structure and importance of the basal cistern has been underlined in the work and by the contributions of the most renowned Turkish neurosurgeon, M. Gazi Yasargil. In order to correctly perform the endonasal approaches to the skull base areas, one needs to understand the same anatomy from the opposite perspective. 